\title{
THE RANGE OF A RING HOMOMORPHISM FROM A COMMUTATIVE $C^{*}$-ALGEBRA
}

\author{
LAJOS MOLNÁR \\ (Communicated by Palle E. T. Jorgensen)
}

\begin{abstract}
We prove that if a commutative semi-simple Banach algebra $\mathcal{A}$ is the range of a ring homomorphism from a commutative $C^{*}$-algebra, then $\mathcal{A}$ is $C^{*}$-equivalent, i.e. there are a commutative $C^{*}$-algebra $\mathcal{B}$ and a bicontinuous algebra isomorphism between $\mathcal{A}$ and $\mathcal{B}$. In particular, it is shown that the group algebras $L^{1}(\mathbb{R}), L^{1}(\mathbb{T})$ and the disc algebra $A(\mathbb{D})$ are not ring homomorphic images of $C^{*}$-algebras.
\end{abstract}

\section{INTRODUCTION}

There are many papers that deal with the beautiful theory of the so-called operator ranges. A subspace of a Banach space is said to be an operator range if it is a continuous linear image of a Banach space (see $[\mathrm{Cro}, \mathrm{FW}]$ and the references therein). It turns out that this condition for a subspace is surprisingly strong. To mention one of the easiest examples which is common in spirit with our results below, we recall that if a Banach space is the range of a continuous linear operator from a Hilbert space, then the Banach space in question is in fact linearly homeomorphic to a Hilbert space. Inspired by these investigations of operator ranges, in our recent paper $[\mathrm{Mol}]$ we started to study similar questions concerning the ranges of ring homomorphisms of certain particular but important Banach algebras. It has been shown that there do not exist surjective ring homomorphisms between different $p$-classes of an infinite-dimensional $H^{*}$-algebra. As a continuation of that work, in this present note we prove the following interesting theorem. If a commutative semi-simple Banach algebra $\mathcal{A}$ is the range of a (possibly discontinuous) ring homomorphism from a commutative $C^{*}$-algebra, then $\mathcal{A}$ is $C^{*}$-equivalent. This means that in spite of the fact that our transformation is far from preserving such essential characteristics of a $C^{*}$-algebra as the involution or the norm, the range still does not forget its $C^{*}$-origin.

Received by the editors November 21, 1994.

1991 Mathematics Subject Classification. Primary 46J05, 46E25.

Key words and phrases. Ring homomorphism, commutative Banach algebra, Gelfand representation.

Research partially supported by the Hungarian National Research Science Foundation, Operating Grant Number OTKA 1652 and K\&H Bank Ltd., Universitas Foundation. 


\section{THE RESUlTS}

Considering the Stone-Weierstrass theorem, our first result, which is fundamental from the view-point of this paper, may seem to be slightly surprising since although self-adjointness and closedness are not assumed for a subalgebra of $C_{0}(X)$, the Banach algebra of all continuous complex-valued functions on the locally compact Hausdorff space $X$ vanishing at infinity, our theorem characterizes $C_{0}(X)$ among its subalgebras (for other characterizations see the excellent survey [Bur]). We recall that a subset $\mathcal{A}$ of $C_{0}(X)$ is called separating if for every $x, y \in X, x \neq y$, there is a function $f \in \mathcal{A}$ such that $f(x) \neq f(y)$. We say that $\mathcal{A}$ vanishes nowhere if for every $x \in X$ there exists an element $f$ of $\mathcal{A}$ for which $f(x) \neq 0$.

Theorem 1. Suppose that $X$ and $Y$ are locally compact Hausdorff spaces. If $\Phi: C_{0}(X) \rightarrow C_{0}(Y)$ is a ring homomorphism whose range contains a separating subalgebra of $C_{0}(Y)$ which vanishes nowhere, then $\Phi$ is a surjective.

Proof. We first show that the kernel $\operatorname{ker} \Phi$ of $\Phi$ is a closed ring ideal of $C_{0}(X)$. To prove the closedness, let $f_{0} \in \overline{\operatorname{ker} \Phi}$. Then for an arbitrary $f \in C_{0}(X)$ there exists an element $g \in \operatorname{ker} \Phi$ such that $\left\|f f_{0}-g\right\|<1$. Let us define

$$
h=\sum_{n=1}^{\infty}\left(f f_{0}-g\right)^{n} \in C_{0}(X) .
$$

We have $h\left(f f_{0}-g\right)=h-\left(f f_{0}-g\right)$ and this implies

$$
\Phi(h) \Phi(f) \Phi\left(f_{0}\right)=\Phi(h)-\Phi(f) \Phi\left(f_{0}\right) .
$$

In particular, $\Phi(f)(y) \Phi\left(f_{0}\right)(y) \neq 1$ for every $y \in Y$. Assume that $\Phi\left(f_{0}\right) \neq 0$, i.e. there is a point $y_{0} \in Y$ such that $\Phi\left(f_{0}\right)\left(y_{0}\right) \neq 0$. The condition posed on the range of $\Phi$ implies that one can choose a function $f \in C_{0}(X)$ with $\Phi(f)\left(y_{0}\right) \Phi\left(f_{0}\right)\left(y_{0}\right)=1$. Thus, we obtain $\Phi\left(f_{0}\right)=0$.

We now assert that every closed ring ideal of $C_{0}(X)$ is in fact an algebra ideal which is a consequence of the existence of an approximating unit. Indeed, let $\lambda \in \mathbb{C}$. By the Urysohn lemma [Rud, 2.12] we infer that for every compact subset $K$ of $X$ there is a function $f_{K} \in C_{0}(X)$ such that $\left|f_{K}\right| \leq|\lambda|$ and $\left.f_{K}\right|_{K}=\lambda$. Since the net $\left(f f_{K}\right)$ converges uniformly to $\lambda f$ for every $f \in C_{0}(X)$, we have the desired statement.

Using [FD, 8.9. Proposition] it follows that there is a closed subset $F$ of $X$ such that

$$
\operatorname{ker} \Phi=\left\{f \in C_{0}(X):\left.f\right|_{F}=0\right\} .
$$

Our next claim is that $\Phi$ can be considered acting on $C_{0}(F)$. To show this, we prove that every continuous function $f$ on the locally compact Hausdorff space $F$ vanishing at infinity can be extended to an element of $C_{0}(X)$. Indeed, let $X_{\infty}=$ $X \cup\{\infty\}$ be the one-point compactification of $X$ which is a compact Hausdorff space. If $\tilde{f}$ is defined on $F \cup\{\infty\}$ as $f$ on $F$ and as 0 at the point $\infty$, then $\tilde{f}$ is a continuous function on a closed subset of a normal space. By the Tietze extension theorem there exists a continuous extension $\tilde{\tilde{f}}$ of $\tilde{f}$ onto the whole space $X_{\infty}$. The restriction of $\tilde{\tilde{f}}$ onto $X$ will posess the desired properties. Hence, if we define $\Psi: C_{0}(F) \rightarrow C_{0}(Y)$ by $\Psi(f)=\Phi(\tilde{f})$ where $f \in C_{0}(F)$ and $\tilde{f} \in C_{0}(X)$ is an 
extension of $f$, then $\Psi$ is a well-defined injective ring homomorphism whose range is equal to that of $\Phi$. Consequently, we may and do assume that our $\Phi$ given in the statement of the theorem is injective.

In the following step we show that for every $y \in Y$ there exist a point $\varphi(y) \in X$ and a ring automorphism $\tau_{y}$ of $\mathbb{C}$ such that

$$
\Phi(f)(y)=\tau_{y}(f(\varphi(y))) \quad\left(f \in C_{0}(X)\right) .
$$

To this end, let $y \in Y$ be fixed for a moment. If $\Phi_{y}: C_{0}(X) \rightarrow \mathbb{C}$ denotes the mapping $f \mapsto \Phi(f)(y)$, then the condition concerning the range of $\Phi$ implies that $\Phi_{y}$ is surjective and the argument above shows that $\operatorname{ker} \Phi_{y}$ is a closed algebra ideal of $C_{0}(X)$ which is regular in the sense of [FD, 5.9. Definition]. Since $\mathbb{C}$ is a simple ring, we have the maximality of the ideal ker $\Phi_{y}$ and hence, by [FD, 8.8. Corollary] there is a point $x=\varphi(y) \in X$ such that

$$
\operatorname{ker} \Phi_{y}=I_{x}=\left\{f \in C_{0}(X): f(x)=0\right\} .
$$

Therefore, the kernels of $\Phi_{y}$ and that of the evaluation map of $C_{0}(X)$ associated with the point $x$ are equal. This implies the existence of a ring automorphism $\tau_{y}$ of $\mathbb{C}$ such that

$$
\Phi_{y}(f)=\tau_{y}(f(x))=\tau_{y}(f(\varphi(y)))
$$

holds for every $f \in C_{0}(X)$.

Our next goal is to prove that the function $\varphi: Y \rightarrow X$ is continuous. Let $\left(y_{\alpha}\right)$ be a net in $Y$ converging to $y \in Y$. Let $\varphi(y) \in U \subset X$ be an open set and suppose that $f \in C_{0}(X)$ vanishes on $X \backslash U$ and $f(\varphi(y))=1$. Since

$$
\tau_{y_{\alpha}}\left(f\left(\varphi\left(y_{\alpha}\right)\right)\right)=\Phi(f)\left(y_{\alpha}\right) \rightarrow \Phi(f)(y)=\tau_{y}(f(\varphi(y)))=1,
$$

there is an index $\alpha_{0}$ such that $f\left(\varphi\left(y_{\alpha}\right)\right) \neq 0$, i.e. $\varphi\left(y_{\alpha}\right) \in U$ holds for every $\alpha \geq \alpha_{0}$. This implies $\varphi\left(y_{\alpha}\right) \rightarrow \varphi(y)$ and we have the continuity of $\varphi$.

Let us show now that $\varphi$ is injective. Suppose, on the contrary, that there are $y, y^{\prime} \in Y$ such that $y \neq y^{\prime}$ but $\varphi(y)=\varphi\left(y^{\prime}\right)$. It follows from (1) that for every function $f \in C_{0}(X)$ which vanishes at $x=\varphi(y)=\varphi\left(y^{\prime}\right)$, we obtain $\Phi(f)(y)=$ $\Phi(f)\left(y^{\prime}\right)=0$. But $\Phi$ is a ring isomorphism from $C_{0}(X)$ onto $r n g \Phi$ and the ideal $I_{x}$ is a maximal ring ideal in $C_{0}(X)$ which is an easy consequence of the simplicity of the ring $\mathbb{C}$. Moreover, it is not hard to see that the ideal of $r n g \Phi$ consisting of all functions from the range which vanish at $y$ and $y^{\prime}$ is properly contained in $I_{y} \cap r n g \Phi$ or in $I_{y^{\prime}} \cap r n g \Phi$. Indeed, if there is a $g \in r n g \Phi$ with $g(y)=0$ and $g\left(y^{\prime}\right) \neq 0$ or with $g(y) \neq 0$ and $g\left(y^{\prime}\right)=0$, then this is obvious. Consequently, assume that $g(y)=0$ or $g\left(y^{\prime}\right)=0$ implies $g(y)=g\left(y^{\prime}\right)=0$ for every $g \in r n g \Phi$. Let $h$ be an element of the separating subalgebra of $r n g \Phi$ such that $h(y) \neq h\left(y^{\prime}\right)$. Hence, $h(y) \neq 0$ and $h\left(y^{\prime}\right) \neq 0$. Then it is elementary that for every $a, b \in \mathbb{C}$ the system of equations

$$
\begin{gathered}
\alpha h(y)+\beta h^{2}(y)=a, \\
\alpha h\left(y^{\prime}\right)+\beta h^{2}\left(y^{\prime}\right)=b
\end{gathered}
$$

can be solved. Now let $a=0$ and let $b=1$. Since $\alpha h+\beta h^{2} \in r n g \Phi$ vanishes at $y$ and does not equal 0 at $y^{\prime}$, we obtain the statement. As a consequence, we 
infer that $\Phi$ maps a maximal ideal into a nonmaximal one and this is an obvious contradiction. Thus, we have the injectivity of $\varphi$.

We next prove that $r n g \varphi$ is dense in $X$. Suppose that there is a point $x \in X$ which does not belong to the closure of $r n g \varphi$. Then by the Urysohn lemma we have a function $f \in C_{0}(X)$ such that $f \neq 0$ and $\left.f\right|_{r n g \varphi}=0$. Using (1), this implies that $\Phi(f)=0$ which contradicts the injectivity of $\Phi$.

Now our claim is that $\tau_{y}$ is continuous for all but a finite number of $y \in Y$. To show this, let $\left(y_{n}\right)$ be a sequence of pairwise different points of $Y$ such that $\tau_{y_{n}}$ is discontinuous (we shall briefly say that $y_{n}$ is a point of discontinuity) for every $n \in \mathbb{N}$. Considering a subsequence of $\left(\varphi\left(y_{n}\right)\right)$ if necessary and using an argument similar to the proof of the statement concerning the cardinality of a perfect set, we may assume that every $\varphi\left(y_{N}\right)$ is an isolated point of $D=\left\{\varphi\left(y_{n}\right): n \in \mathbb{N}\right\}$. If $\tau$ is a discontinuous automorphism of $\mathbb{C}$, then $\tau$ maps every disc onto an unbounded set [Kuc, Theorem 2, p. 360]. Consequently, we can choose a sequence $\left(\lambda_{n}\right)$ of complex numbers converging to 0 such that $\left|\tau_{y_{n}}\left(\lambda_{n}\right)\right| \rightarrow \infty$. Let us define $f: \bar{D} \rightarrow \mathbb{C}$ by

$$
f(x)=\left\{\begin{array}{lll}
\lambda_{n} & \text { if } \quad x=\varphi\left(y_{n}\right), n \in \mathbb{N}, \\
0 & \text { if } \quad x \in \bar{D} \backslash D .
\end{array}\right.
$$

It is easy to see that $f \in C_{0}(\bar{D})$ and hence there is an extension $\tilde{f} \in C_{0}(X)$ of $f$. Since

$$
\Phi(\tilde{f})\left(y_{n}\right)=\tau_{y_{n}}\left(\tilde{f}\left(\varphi\left(y_{n}\right)\right)\right)=\tau_{y_{n}}\left(\lambda_{n}\right) \quad(n \in \mathbb{N})
$$

and the right side is not bounded, we arrive at a contradiction.

Let $\left\{y_{1}, \ldots, y_{n}\right\}$ be the set of all points of discontinuity. We assert that $y_{1}, \ldots, y_{n}$ and $\varphi\left(y_{1}\right), \ldots, \varphi\left(y_{n}\right)$ are isolated points of $Y$ and $X$, respectively. To see this, suppose that $\left(y_{\alpha}\right)$ is a net in $R=Y \backslash\left\{y_{1}, \ldots, y_{n}\right\}$ converging to $y_{1}$. It is well known that there are exactly two continuous automorphisms of $\mathbb{C}$, namely, the identity and the conjugation [Kuc, Lemma 1, p. 356]. Therefore, by (1) we have

$$
\left|f\left(\varphi\left(y_{\alpha}\right)\right)\right|=\left|\tau_{y_{\alpha}}\left(f\left(\varphi\left(y_{\alpha}\right)\right)\right)\right| \longrightarrow\left|\tau_{y_{1}}\left(f\left(\varphi\left(y_{1}\right)\right)\right)\right|
$$

and

$$
\left|f\left(\varphi\left(y_{\alpha}\right)\right)\right| \longrightarrow\left|f\left(\varphi\left(y_{1}\right)\right)\right|
$$

for every $f \in C_{0}(X)$. These imply that $\left|\tau_{y_{1}}(\lambda)\right|=|\lambda|$ holds for every $\lambda \in \mathbb{C}$, i.e. $\tau_{y_{1}}$ is continuous which is a contradiction. Thus, $y_{1}$ is an isolated point of $Y$. By the Urysohn lemma there is a function $f \in C_{0}(X)$ such that $f\left(\varphi\left(y_{i}\right)\right)=1(i=1, \ldots, n)$. Then the function $y \mapsto \Phi(f)(y)=\tau_{y}(f(\varphi(y)))$ is continuous and vanishing at infinity. Let us consider it only on the closed set $R$. Since this restriction also vanishes at infinity, we have a compact set $K \subset R$ such that $|f(\varphi(y))|<1 / 2$ $(y \in R \backslash K)$. Consequently, $\varphi(K)$ is a compact subset of $\varphi(R)$ with the property that $|f(x)|<1 / 2$ for every $x \in \varphi(R) \backslash \varphi(K)$. This implies that $\varphi\left(y_{1}\right), \ldots, \varphi\left(y_{n}\right)$ are not accumulation points of $\varphi(Y)$. Using the density of $r n g \varphi$ in $X$ we infer that these points are isolated in $X$.

Let $S=X \backslash\left\{\varphi\left(y_{1}\right), \ldots, \varphi\left(y_{n}\right)\right\}$. If $f \in C_{0}(S)$, let

$$
\Psi(f)(y)=\tau_{y}(f(\varphi(y)))(y \in R) .
$$


Then there exists an $\tilde{f} \in C_{0}(X)$ such that $\tilde{f} \mid S=f$ and hence $\Psi(f)=\Phi(\tilde{f}) \mid R$. Since $R$ is closed in $Y$, we have $\Psi(f) \in C_{0}(R)$. The range of $\Phi$ contains a subalgebra of $C_{0}(Y)$ which separates the points of $Y$ and vanishes nowhere. Let $\mathcal{A}$ denote the set of the restrictions of the elements of this subalgebra onto $R$. It is easy to see that $\overline{\Psi(f)}=\Psi(\bar{f})\left(f \in C_{0}(S)\right)$, i.e. the range of $\Psi$ is self-adjoint. The family of all functions from $r n g \Psi$ which are of the form

$$
\sum_{i} \alpha_{i} g_{i}+\sum_{j} \beta_{j} \overline{h_{j}}+\sum_{l} \gamma_{l} g_{l} \overline{k_{l}} \quad\left(g_{i}, h_{j}, k_{l} \in \mathcal{A}\right)
$$

is a subalgebra satisfying the assumptions of the Stone-Weierstrass theorem. This implies the density of $r n g \Psi$ in $C_{0}(R)$. Since $\varphi(R)$ is dense in $S$, we obtain that $\Psi$ is an isometry and, consequently, we have $r n g \Phi \mid R=r n g \Psi=C_{0}(R)$.

Finally, if $g \in C_{0}(Y)$ is arbitrary and $f \in C_{0}(S)$ is such that $\Psi(f)=g \mid R$, then let the function $\tilde{f}: X \rightarrow \mathbb{C}$ be defined by

$$
\tilde{f}(x)=\left\{\begin{array}{lll}
\tau_{y_{i}}^{-1}\left(g\left(y_{i}\right)\right) & \text { if } \quad x=\varphi\left(y_{i}\right) \quad(i=1, \ldots, n), \\
f(x) & \text { if } \quad x \in S .
\end{array}\right.
$$

It is easy to see that $\tilde{f} \in C_{0}(X)$ and $\Phi(\tilde{f})=g$. This completes the proof.

Using the language of Banach algebras we can reformulate the statement of the previous theorem in the following way.

Theorem 2. Let $\mathcal{A}$ be a commutative $C^{*}$-algebra and let $\mathcal{B}$ be a commutative semisimple Banach algebra. If $\Phi: \mathcal{A} \rightarrow \mathcal{B}$ is a ring homomorphism whose range contains a dense subalgebra of $\mathcal{B}$, then $\Phi$ is surjective and $\mathcal{B}$ is $C^{*}$-equivalent.

Proof. Let $\hat{\mathcal{A}}$ and $\hat{\mathcal{B}}$ be the structure spaces of $\mathcal{A}$ and $\mathcal{B}$, respectively, and let $\mathcal{G}_{\mathcal{A}}$ and $\mathcal{G}_{\mathcal{B}}$ denote the corresponding Gelfand representations. Then $\Psi=\mathcal{G}_{\mathcal{B}} \circ \Phi \circ$ $\mathcal{G}_{\mathcal{A}}{ }^{-1}$ is a ring homomorphism from $C_{0}(\hat{\mathcal{A}})$ into $C_{0}(\hat{\mathcal{B}})$. Clearly, $r n g \Psi$ contains a dense subalgebra. Since the range of the Gelfand representation is separating and vanishing nowhere, the same holds for its subalgebra in question. Now, Theorem 1 implies that $\Psi$ is surjective and then the same holds for $\Phi$ as well. Finally, the continuity of the Gelfand representation and the Banach theorem on the bounded inverse yield the $C^{*}$-equivalence of $\mathcal{B}$ to $C_{0}(\hat{\mathcal{B}})$.

As an application of the results above we prove the following corollary.

Corollary. The group algebras $L^{1}(\mathbb{R}), L^{1}(\mathbb{T})$ and the disc algebra $A(\mathbb{D})$ are not ring homomorphic images of commutative $C^{*}$-algebras.

Proof. Examining the proof of our Theorem 2 we obtain that it is sufficient to prove that the Gelfand representations of these Banach algebras are not surjective. But this is a consequence of the Gelfand theory and the well-known results [Rud, Chapter 9, Exercise 2], [Rud, 5.15 Theorem] and [Pal, 3.2.13].

To conclude the paper, we note that in the light of Cuntz's fundamental theorem on locally $C^{*}$-equivalent algebras [Cun], it would also be interesting to study the noncommutative counterparts of our Theorem 2. 


\section{REFERENCES}

[Bur] R.B. Burckel, Characterization of $C(X)$ among Its Subalgebras, Lecture Notes in Pure Appl. Math. 6, Marcel Dekker, 1972. MR 56:1068

[Cro] R.W. Cross, On the continuous linear image of a Banach space, J. Austral. Math. Soc. (Series A) 29 (1980), 219-234. MR 81f:47001

[Cun] J. Cuntz, Locally $C^{*}$-equivalent algebras, J. Funct. Anal. 23 (1976), 95-106. MR 56:6398

[FD] J.M.G. Fell and R.S. Doran, Representations of *-Algebras, Locally Compact Groups, and Banach *-Algebraic Bundles, Vol. I., Academic Press, 1988. MR 90c:46001

[FW] P.A. Fillmore and J.P. Williams, On operator ranges, Adv. Math. 7 (1971), 254-281. MR 45:2518

[Kuc] M. Kuczma, An Introduction to The Theory of Functional Equations and Inequalities, Państwowe Wydawnictwo Naukowe, Warszawa, 1985. MR 86i:39008

[Mol] L. Molnár, Algebraic difference between p-classes of an $H^{*}$-algebra, Proc. Amer. Math. Soc. (to appear). CMP 94:17

[Pal] T. W. Palmer, Banach Algebras and The General Theory of *-Algebras, Vol. I., Encyclopedia Math. Appl. 49, Cambridge University Press, 1994. MR 95c:46002

[Rud] W. Rudin, Real and Complex Analysis, Tata McGraw-Hill Publishing Co. Ltd., New Delhi, 1983.

Institute of Mathematics, Lajos Kossuth University, 4010 Debrecen, P.O.Box 12, HUNGARY

E-mail address: molnarl@math.klte.hu 